\title{
A novel collection technique for the improvement of semen quality
}

\begin{abstract}
To date, the common thread in the use of semen extenders/collection techniques, whether they are being used for fresh extended semen, chilled semen, or cryopreserved semen, is that the extenders have all traditionally been added post-collection. The objective of this study was to determine if modifying the method of collection/ extension of semen to include a warmed media environment would improve semen parameters by lessening cold and $\mathrm{pH}$ shock. Ten canine semen samples were collected with a modified artificial vagina to allow for a true split collection into to collection containers at one time. The treatment half of the sample was collected into a measured amount of warmed extension media. The control half was collected into a dry container and no attempts to maintain temperature was used. Standard semen parameters, available sperm pool, and number of inseminations were evaluated at specific time intervals and evaluations continued until samples reached zero percent motility. Data analysis was performed with SPSS using the general linear model and appropriate t-tests. There was a difference between the treatment and control groups for Motility $(\mathrm{P}<.001)$, Motility by Time $(\mathrm{P}<.001)$, Time to Zero Motility $(\mathrm{P}<.001)$, Time to Last Full Insemination $(\mathrm{P}<.03)$, Forward Progression $(\mathrm{P}<.001)$, Acrosome Reaction $(\mathrm{P}<.001)$, Acrosome Reaction by Time $(\mathrm{P}<.02)$ and Viability $(\mathrm{P}<.001)$. There was no difference in morphology between the treatment and control groups $(\mathrm{P}>.05)$. Modification of the semen collection/extension procedure resulted in improved semen parameters for extended time-periods post-collection. The data suggest the described collection technique can yield significantly more motile sperm by placing the sample into a physiologically favorable environment (eliminating $\mathrm{pH}$ and cold shock and allowing osmoregulation to begin), thus providing more available sperm for breeding.
\end{abstract}

Volume I Issue I - 2014

\author{
Dustie L Johnson,' Samuel D Prien ${ }^{2,3}$ \\ 'Reproductive Solutions, USA \\ ${ }^{2}$ Department of Obstetrics and Gynecology, Texas Tech \\ University Health Sciences Center, USA \\ ${ }^{3}$ Department of Animal and Food Sciences, Texas Tech University, \\ USA
}

Correspondence: Sam D Prien, Department of Obstetrics and Gynecology, Texas Tech University Health Sciences Center, Lubbock,TX 79430, Texas, USA, Tel 806743 2358, Fax 806743 4238,Email samuel.prien@ttuhsc.edu

Received: June 28, 2014 | Published: July 03, 2014

Keywords: artificial insemination, canine, semen collection

Abbreviations: AI, artificial insemination; GLM, general linear model; SPSS, statistical program for the social sciences

\section{Introduction}

Since artificial insemination's (AI) inception, in the 1300's, and its first documented use in the 1780's, AI has continued to be developed as a tool in both animal and human reproduction. ${ }^{1}$ Realizing early on that raw, unprocessed semen lost fertilizing capability rapidly after ejaculation, it was discovered that the collected semen had to be used the same day (and in cases such as the dog, almost immediately in order to achieve good results- i.e. pregnancy). In order for AI to develop to its full potential, a method had to be discovered to preserve semen for use at a later date.

The first successful, and by far still the most common, way to preserve semen for later use was with semen extenders. ${ }^{2}$ These extenders were created in an attempt to hold sperm in a favorable environment for cellular survival while biochemically placing the cells in suspended animation (delaying their progression toward final maturity) until time for their use. Semen extenders provide nutrients for sperm metabolism, carry additives such as antibiotics and cryoprotectants (for storage at lower temperatures), and provide multiple breedings from one semen sample. Extended semen can be maintained for days (times vary depending on the species) and with the addition of a cryoprotectant, extended semen can also be frozen and remain viable (i.e., produce a pregnancy) for up to 20years. ${ }^{3}$
The common thread with the use of semen extenders and collection techniques is that the extenders have traditionally been added postcollection. While this method does work, it may not be the most efficient method of extension. Sperm are especially susceptible to changes in temperature and $\mathrm{pH} .{ }^{48}$ This shock to the sperm can result in loss of motility and fertilizing capability, rendering it virtually useless in a matter of a few minutes to a few hours. In some species, such as the canine, zero percent motility can be reached in less than one hour.

While attempts are sometimes made to warm the collection container, the containers are still dry. ${ }^{9}$ In theory, protecting semen from both temperature and $\mathrm{pH}$ shock upon collection would extend the functional life and fertilizing capability of the spermatozoa. To test this theory, canine semen was collected in a split ejaculate. Half of the semen was collected using standard techniques, while the other half was collected directly into a measured amount of warmed extender media, in an attempt to lessen or prevent temperature and osmotic shock to the spermatozoa and preserve fertilizing capability.

\section{Materials and methods}

In this experiment, extension of the functional life span and fertilizing capability of semen was attempted through a modification of the traditional collection/extension method. Using the canine as a model (ACUC approved), the quality of semen collected into a measured amount of warmed extension media $(\sim 20 \%$ by volume of the expected volume of ejaculate $=1 \mathrm{ml}$ ) was compared to samples 
collected using traditional methodology. The volume of media chosen should allow for sufficient temperature and $\mathrm{pH}$ protection without causing undo osmotic stress to the sperm. ${ }^{10}$

Collection of semen samples was done using digital manipulation and a modified artificial vagina. ${ }^{11}$ Or this experiment, it was necessary to collect semen so that both the control and treatment samples were collected simultaneously from the animal. This method of collection was necessary in order to eliminate the variation that can be seen between different collections, or even different collection fractions (traditional split collection), within the same animal. No traditional collection device allowed for this type of collection. Therefore, it was necessary to develop a collection device that provided a true split collection (patent awarded).

Prior to collection, the centrifuge tubes used to collect the semen were prepared. The control tube was left dry and no special procedures were taken. The treatment tube had a measured amount $(1 \mathrm{ml})$ of $37^{\circ} \mathrm{C}$ semen extender placed into the tube and this tube continued to be maintained at the $37^{\circ} \mathrm{C}$ temperature throughout the collection process. The extender used in these experiments was Androhep ${ }^{\mathrm{TM}}$ (Minitube of America, Inc.; Verona, WI), a commercial semen extender designed for use in the porcine industry, but found effective in this laboratory for extension of canine semen.

Once the dog had finished ejaculating, the AV was removed. The extension of the treatment sample was then completed immediately using a 2:1 extender to semen ratio by volume. The control sample was held fifteen minutes post-collection before extending at the same ratio. After one hour, both samples were chilled to $5^{\circ} \mathrm{C}$ and stored.

A selected group of semen parameters were evaluated at zero, one, six, twelve, eighteen, and twenty-four hours post-collection and at twenty-four hour intervals thereafter, until zero percent motility was reached. For evaluations conducted after the first hour, a small amount $(\sim .25 \mathrm{ml})$ of semen was removed and warmed in a $37^{\circ} \mathrm{C}$ water bath for five minutes prior to evaluation.

The parameters evaluated included: volume, concentration, motility, forward progression, acrosome reaction, viability, and morphology. All standard semen parameters (volume, concentration, forward progression, viability and motility) were evaluated on a Nikon Alphapot microscope equipped with phase optics (Nikon Inc.; NY, NY) using well documented techniques described for the canine. ${ }^{12}$

In addition to the standard semen parameters, a sample was prepared at each time point to evaluate the percentage of spermatozoa that had undergone the acrosome reaction as an indicator of cells that have begun the process of capacitating. ${ }^{13}$ Evaluations were made after preparing the cells using the chlorotetracycline stain technique of Keel and Webster and examining them using a Zeiss Standard microscope equipped with fluorescence (Carl Zeiss Inc., NY, NY). ${ }^{14}$ The cells were examined using a $520 \mu \mathrm{m}$ excitation filter and a $570 \mu \mathrm{m}$ barrier filter. The intact acrosome cap appeared a fluorescent yellow while the acrosome reacted cells had lost their acrosome cap and therefore appears faded.

Morphology was recorded at the initial time point, approximately one-half way through the evaluations, and when zero percent motility was reached, for both the treatment and control samples by three investigators, to verify that morphology did not change due to treatment. Slide were prepared using a standard Hematoxylin-Eosin stain technique followed by bright-field examination of 100 cells using a 100x oil immersion lens, and noting head, mid-piece and tail defects.
A total of ten ejaculates from ten different animals were used in the study. Because each animal served as its own control, the study was designed as a split-plot with repeated measures having a specific end point. All data were analyzed using the Statistical Program for the Social Sciences (SPSS version 8.0; Chicago, IL). Comparisons between treatment and control groups for motility, acrosome reaction, viability, and morphology were analyzed using the General Linear Model (GLM). Motility at any specific time point, time to zero motility, and time to last full insemination were compared between the treatment and control groups using paired t-tests. Chi-square analysis was used for comparison of the forward progression data.

\section{Results}

As expected, the concentrations of the control $\left(85.2 \times 10^{6} \pm 3.4 \times 10^{6}\right)$ and treatment $\left(87.7 \times 10^{6} \pm 15.0 \times 10^{6}\right)$ groups were similar and remained constant over the course of the experiment $(\mathrm{P}<.481)$. There was no difference in morphology between the treatment and control groups $(\mathrm{P}>.062)$. While there was a difference in morphology over time $(\mathrm{P}<.001)$, this difference was only in tail abnormalities and was expected with the loss of motility and changes associated with cell death. Further, there was also a difference between the three investigators $(\mathrm{P}<.002)$. However, each investigator found no difference between treatment and control groups.

There was a difference in motility due to time $(\mathrm{P}<.001)$, as expected. In addition there was an overall difference between treatment and control groups for motility $(\mathrm{P}<.001)$. As shown in Figure 1, the difference in treatment by time versus control by time was significant $(\mathrm{P}<.001)$. In addition, spermatozoa in the treatment group had significantly higher motility compared to the control at all time-points past the initial evaluation up to 192hours, where there were too few animals for an accurate analysis $(\mathrm{P}<.001)$.

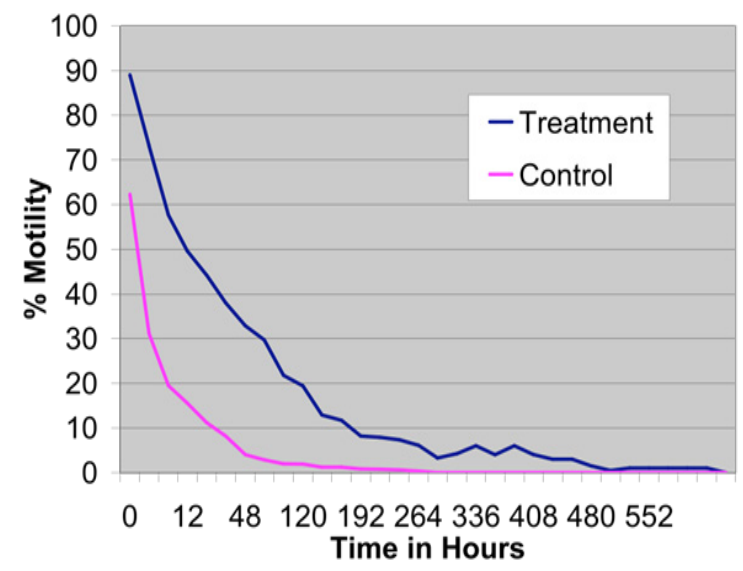

Figure I Improved spermatozoa motility over time of extended canine semen samples collected using a novel physiologically balanced collection technique verses standard procedures $(\mathrm{P}<.00 \mathrm{I})$.

It was possible to determine the time it took for all samples to reach $0 \%$ motility. While the average time to zero percent motility for the control group was 129.6hours (std. error+/-30.75) with a range of 12 to 288 hours, the average time to zero percent motility for the treatment group was 276 hours (std. error+/-49.74) with a range of 48 to 600 hours. This represents a $378 \%$ increase in spermatozoa survival time in the treatment group over the control (Figure 2; $\mathrm{P}<.001$ ).

These findings were confirmed by live/dead staining for viability, which demonstrated greater cell survival in the treatment versus the control at all time-points (Figure 3; $\mathrm{P}<.001$ ). Further, using a five point 
scale, forward progression was significantly higher in the treatment group as compared to the control from the one hour observation point until the end of the experiment (Figure 4; $\mathrm{P}<.001$ ).

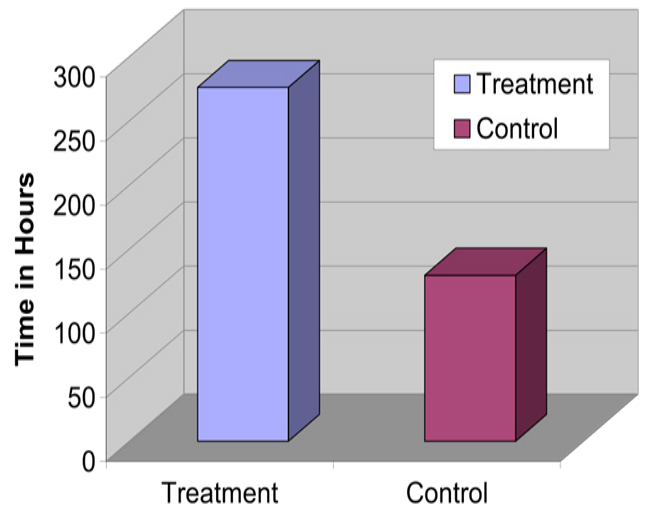

Figure 2 Extended functional life-span (measure as time to $0 \%$ motility) of canine semen samples collected using a novel, physiologically balanced collection technique verses standard procedures (Mean+STD; P<.00I)

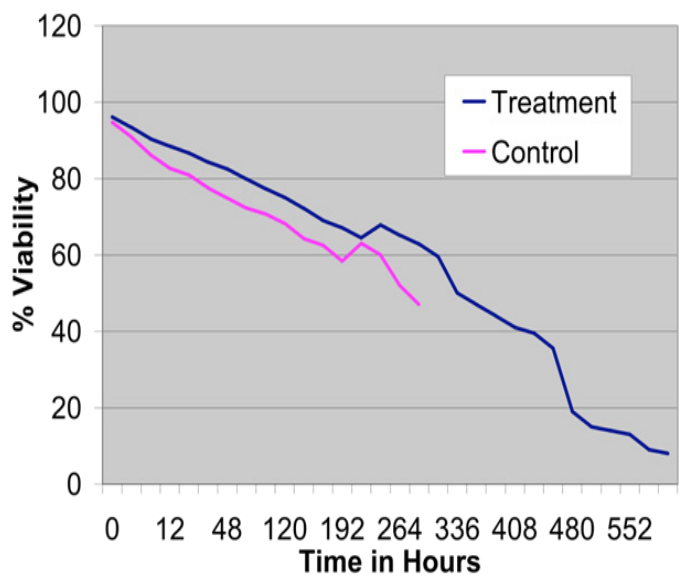

Figure 3 Maintenance of cell viability over time of extended canine semen samples following collection using a novel, physiologically balanced collection technique verses standard procedures $(\mathrm{P}<.00 \mathrm{I})$.

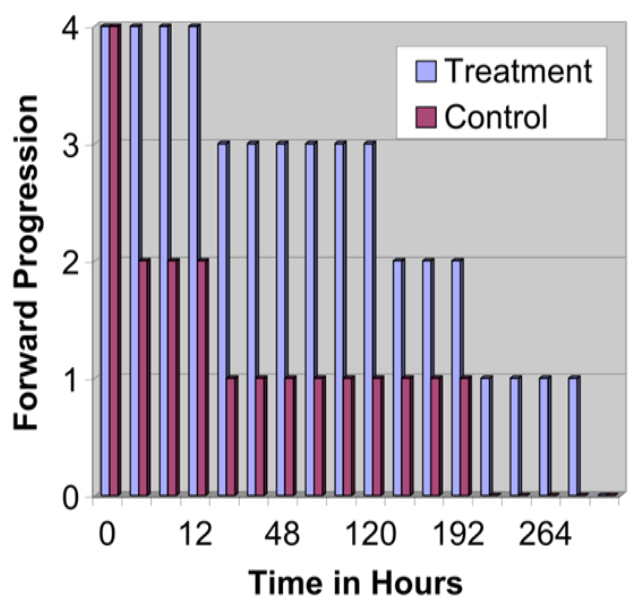

Figure $4 \mathrm{~A}$ comparison of the maintenance of spermatozoa forward progression (measured manually using a 5 point scale) over time of extended canine semen samples collected using a novel, physiologically balanced collection technique verses standard procedures (Mean+STD; $\mathrm{P}<.00 \mathrm{I}$ ).
Finally, on average, it took less than eighteen hours for half of the cells in the control group to undergo the acrosome reaction as determined by fluorescent staining. By contrast, it took approximately sixty hours for half of the cells in the treatment group to stain as acrosome reacted (Figure 5; $\mathrm{P}<.02$ ).

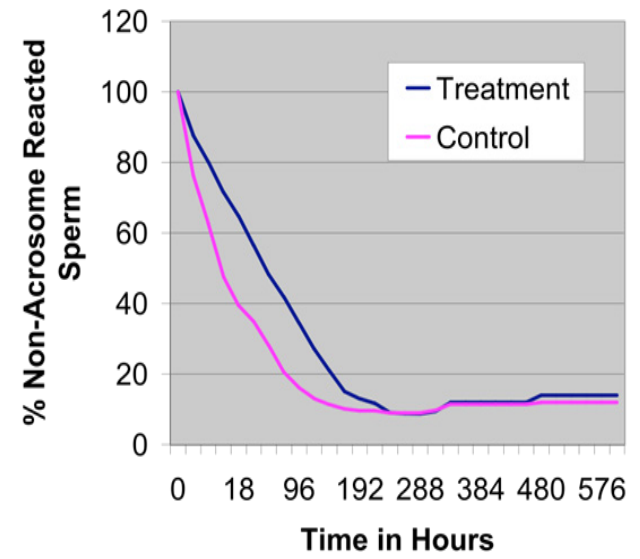

Figure $\mathbf{5}$ The maintenance of non-acrosome reacted spermatozoa over time of extended canine semen samples collected using a novel, physiologically balanced collection technique verses standard procedures $(P<.02)$.

\section{Discussion}

Artificial insemination has been used for several centuries. However, it was not until the early 1900's, when semen extenders were developed, that semen could be stored and used at a time other than immediately after collection. The traditional method of extending semen was, and still is, to add the extender anywhere from a few minutes up to one hour post-collection. While this method does help preserve semen for use at a later time post-collection, data from this experiment suggests it is not the most efficient method.

Previous research has shown spermatozoa to be susceptible to shifts in temperature and $\mathrm{pH}$ (Grootegoed Energy metabolism of spermatids: a review. ${ }^{5,15}$ In theory, collecting semen directly into a measured amount of warmed extender would maintain the semen at a constant temperature and prevent cold shock to the spermatozoa. Further, the spermatozoa would also come in contact with the buffers of the extender immediately upon collection, which helps to prevent shifts in $\mathrm{pH}$ and allows osmoregulation to begin..$^{10}$ Data from the present experiment would appear to support this theory, as all functional semen parameters were improved when the sample was collected directly into the warmed extender.

This novel modification to the collection/extension of semen allows for improved preservation of spermatozoa over time when compared to traditional methods. From the data analysis, it is clear that collecting semen into warmed extender media improved the semen parameters evaluated. Specifically, the functional life span of the spermatozoa, measured as viability, motility, and forward progression and acrosome intact cells was significantly increased in the treatment group as compared to the control.

These improved semen parameters would have several implications for an artificial insemination program. First, collection into warmed extender appeared to improve semen parameters immediately after collection, suggesting previously marginal or unacceptable samples might now be acceptable for use in on-site breeding programs. Second, increased functional semen parameters in a "healthy" donor would 
suggest a greater number of inseminations upon collection and these donors could maintain at least one full insemination dose much longer due to the treatment. Further, times to full acrosome reaction were delayed in the treatment group. By maintaining a greater percentage of motile (and therefore viable), non-acrosome reacted sperm, the treatment group maintained a full insemination dose for a significantly greater length of time as compared to the control. These data suggest that a simple modification in collection procedures might improve the results of programs that collect and ship extended semen over great distances for use in breeding programs. The results of this experiment also suggest that this method would be helpful in maintaining semen parameters in cryopreserved (frozen) semen. Given these findings, experiments are currently underway with a modified specimen collection device which;

i. Minimizes the exposed sample surface area

ii. Minimizes the surface area to volume ratio

iii. Thermal insulates the sample

iv. Contains the measured media content to control $\mathrm{pH}$ and limit osmotic shock

\section{Acknowledgements}

The authors wish to thank Dr. Carl Clary for his assistant in animal collection.

\section{Conflict of interest}

Author declares that there is no conflict of interest.

\section{References}

1. Spallanzani L, Dalyell JG. Tracts on the Natural History of Animals and Vegetables. (Translated title). 2nd ed. London: Creech and Archd, Constable Edinburgh. 1803.

2. Bearden HJ, Fuquay JW. Applied Animal Reproduction. 5th ed. New Jersey, USA: Prentice-Hall Inc; 2000.
3. Phillips PH, Lardy HA. A yolk-buffer pabulum for the preservation of bull semen. J Dairy Sci. 1940;23(5):399-404.

4. Drobnis EZ, Crowe LM, Berger T, et al. Cold shock damage is due to lipid phase transitions in cell membranes: a demonstration using sperm as a model. J Exp Zool. 1993;265(4):432-437.

5. Grootegoed JA, Boer PJD. Energy metabolism of spermatids: a review. In: Hamilton DW, Waites GHM, editors. Cellular and molecular events in spermiogenesis. Cambridge University Press; 1987. p. 193-216.

6. Plummer JM, Watson PF. Ultrastructual localization of calcium ions in ram spermatozoa before and after cold shock as demonstrated by a pyroantimonate technique. J Reprod Fertil. 1985;75(1):255-263.

7. Tao J, Junying D, Kleinhans FW, et al. The effect of collection temperature, cooling rate and warming rate on chilling injury and cryopreservation of mouse spermatozoa. J Reprod Fertil. 1995;104(2):231-236.

8. White IG. Lipids and calcium uptake of sperm in relation to cold shock and preservation: a review. Reprod Fertil Dev. 1993;5(6):639-658.

9. Knox R, Levis D, Safranski T, et al. An update on North American boar stud practices. Theriogenology. 2008;70(8):1202-1208.

10. Strzezek R, Fraser L. Characteristics of spermatozoa if whole ejaculate and sperm-rich fraction of dog semen following exposure to media varying in osmolality. Reprod Biol. 2009;9(2):113-126.

11. Christensen BW, Asa CS, Wang C, et al. Effect of semen collection method on sperm motility of grey wolves (Canis lupus) and domestic dogs (C. I. familiaris). Theriogenology. 2011;76(5):975-980.

12. Bardin CW, Chenge CY, Musto NA, et al. The Sertoli Cell. In: The Physiology of Reproduction. New York: Raven Press Ltd. 1988. p. 933-974.

13. Farstad W. Customizing semen preservation protocols for individual dogs and individual species: sperm preservation beyond the state of the art. Reprod Domest Anim. 2012;47(Suppl 6):269-273.

14. Keel BA, Webster BW. Handbook of the Laboratory Diagnosis and Treatment of Infertility. Boca Raton, Florida: CRC Press Inc; 1990.

15. Seager SWJ, Fletcher WS. Collection, storage, and insemination of canine semen. Lab Anim Sci. 1972;22(2):177-182. 